$N \cup \cup H$ I $\cup$ ᄃ $\cup \cdots$

O I E U I A F E O N G I UAMUח , -

U F M Y B B HMGRLBDPBDLRBEFBA ।

$U D H P$ H Y D R A U L I C S L OWG TRA UBAFVNK,

J A TRUAOLONOXUBRGHZFGDNLIERNGM J b

A MOET S U D U Y G E A H N I O Z P X O KP SWQRDSA U,

EK J I CKBZ J GR D CK I O PMNE SWLNCAWZ YKFEQLO,

$M O T C O O L I N G D S Q O M G D N V U S G R V L G R A K G E C L Z E M S$,

TNUSVCESOPODINGRE E O N Y A Z T ENNAXIRCNIFZKM!,

$D C O J Q Y O H I N M N V C S E Y L I N F W C H M G R X B D \longrightarrow L D B E U B A \vdash$



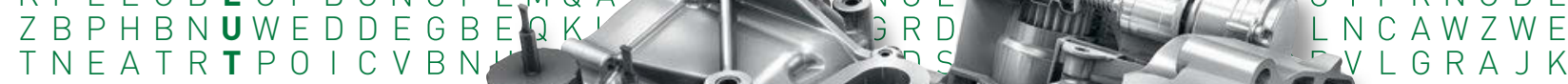

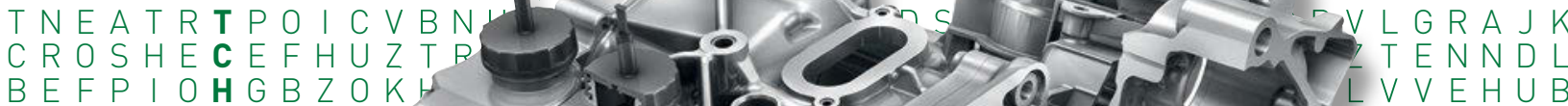
SOBWREQR I UN J R P FE I A YWTRDXZT O JUHBZGLMOE S $D G J L Y C B M N S G N$ E T UOMBCLZKOA J PMK I W P M SW Q V G T D A E W T R D I Z $A T Z M \mid T J H L M G H A M$ LWMTNOGLETGFDSin:?

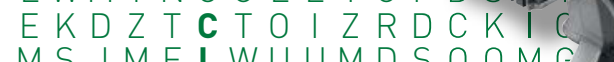
$M S J M E L W U U M D S Q O M G$ TKJ D G UKP SDGD I NG JNUJ | CKA I J CWQY J A NCOTEHODKTOBNJORY $A Y \mid U R K N$ I SLW I UARN $U J K O T L C A T P M E B D P E$ A A O A X I A L Q DM I UAR 15 $M D M K S N J R B U S Q O M G$ ? UAOOS I ANMIKIBDPBQ M. 1.04 G FOTMENDBUTETREWQL\&AMMB CXYMLMOKN I J B HUZGF CDMONGANNOIUMDEA INHFO $80 M P N A E F V E G B Z H N U J M \mid K O Q A$ PEKTS I O GATAN I JUHBZGVTFCRDXESNWASRECVFHKNUTEQTF CGTY I ZSRDC I ADGJLYCBMWRZIPSFHKTVNZLMO I JEUHBZGWR $J T Z M K I H G F D W Q E T U O M B C Y N \vee X A D G J L K H E S Y S C B F G M H T I L Q N V$ 


\section{Wet Double Clutch: Thinking in Systems}

\author{
Andreas Englisch \\ Andreas Goetz \\ Andreas Baumgartner \\ Thomas Endler \\ Christian Lauinger \\ Stefan Steinmetz
}

D v v

$\mathrm{J} \mathrm{ZH}_{L}$

$A G Q S W \mid\llcorner\cdots$

$F \mid M B C H S E H \in\llcorner$

I C E B STPOIODCVト O

$D G \vee T Q U J Z R E L K J H G F D S A \mid v_{1} v_{1}$

Y $Y$ M T X A G Y P H C E A YWS X E CK,

$C X \vee N H O U B I J B Z G V F C R D E S N W A S K$

$Z V T F L U J A D G Y C B M W P Z$ I P S F K T V N Z L MU,

X D B P R UTETMBCYNVXADG J L K H E Y S C B M B L

DC S KUPOWRWZTWHNEDKUNWPONCALVIKZTWHI

EHKLPFLKJKOIUZTREWQYXCVBNMIQWUOIUZTRt


I Z Z KOG I KCKPMNESWLNCXWZYKFEDIOPPMNESWLNCX

$R \cup C Z G Z M Q G O D N V U S G R V L G R V K G E C E Z E M D N V \cup S G R V L G$ QATSLOKZINEXOMNYAZTEWNFXJLRNIFEXOMNYAZTEW

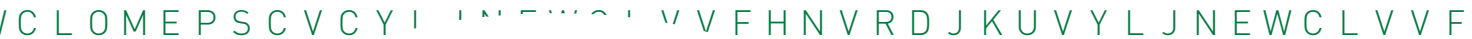
$F A M \cup A N J Y \cap \cdots$

KMNSRDO

L I E P N

B S A T B

$P \mid E P$ I

$R \cup C Z$

B S A

D G V

$Y \perp \Lambda$

C X i

Z V

$X \mathrm{D}$
.1 I O IN IN N L I EK.

D D L R B F B A F V N K

. UAH | OGDNO | ERNGM

OQ O D N V U S R V L G R VKG

PDBDDLRBEFBAFVNKFN

, OTRELK J H G F D S AMMBVCX $X A Z Y W P H C E Q A Y W S X E E C R F$ I $O U B \mid J B Z G V T F C R D X E S N W A S$ U J RDG Y C B MWRZ I PSFHKTVN RUTETMBCYNVXADGJLKHES P OWRWZTWHNEDKUNWPONCA $F L K J K O I U Z T R E W Q Y X C \vee B N M$ HASESVNPIZRWQSCGZNJIM G I KCKPMNESWLNCXWZ Y KFF I A S U S V N P I ZRWQ S C G Z N J I I KCKPMNESWLNCXWZYKF ' OX ODNVUSGRVLGRVKG

1 I NEXOMNYAZTEWNF.

' CYL JNEWCLVVFH' $\cap N \vee \cup S G R \vee L G F$


ZMFDRODFNGLDFN $\therefore A \cup K Z Q H|O G D N O| E R N$ I REWSPDLRBEFBAFVNK $L K Z Q H|O G D N O| E R N G N$ Z E M D N U S G R V L G R V K NSPDLRB E F B F V N K F MOLK JHGFDSAMMB V B Z P H C E Q A YW S X E C $\cong \vee B Z G V T F C R D X E S N$ 10 Y C B MWR Z I P F H K $B M B C Y N \vee X A D G J L K$ KZTWHNEDKUNWPr 


\section{Introduction}

\section{Status}

In ten years, the number of wet and dry double clutch transmissions (DCT) will make up approximately $20 \%$ of the total automatic transmission market.

Against this backdrop, customers are faced with the question of which double clutch system is right for their application. Both dry and wet double clutches have proven themselves in volume production. There are various designs and various forms of actuation within the two systems, with key differences being in the torque capacity, space requirements, weight and inertia of masses [1].
A whole range of wet double clutch systems have since been developed by LuK in order to be able to serve a vast array of applications. The first wet double clutch went into volume production in 2013.

In addition to the actual double clutches, other components such as dampers, centrifugal pendulum-type absorbers and actuators are also available. The focus is on a perfectly matched overall system that meets the target parameters of comfort, consumption and costs in the best possible manner. To do this, components and assemblies need to be standardized to pool volumes and thus be able to continue to offer appealing solutions in the future.

Throughout the development phase, various different concepts were analyzed and compared on a broad basis. The clutch

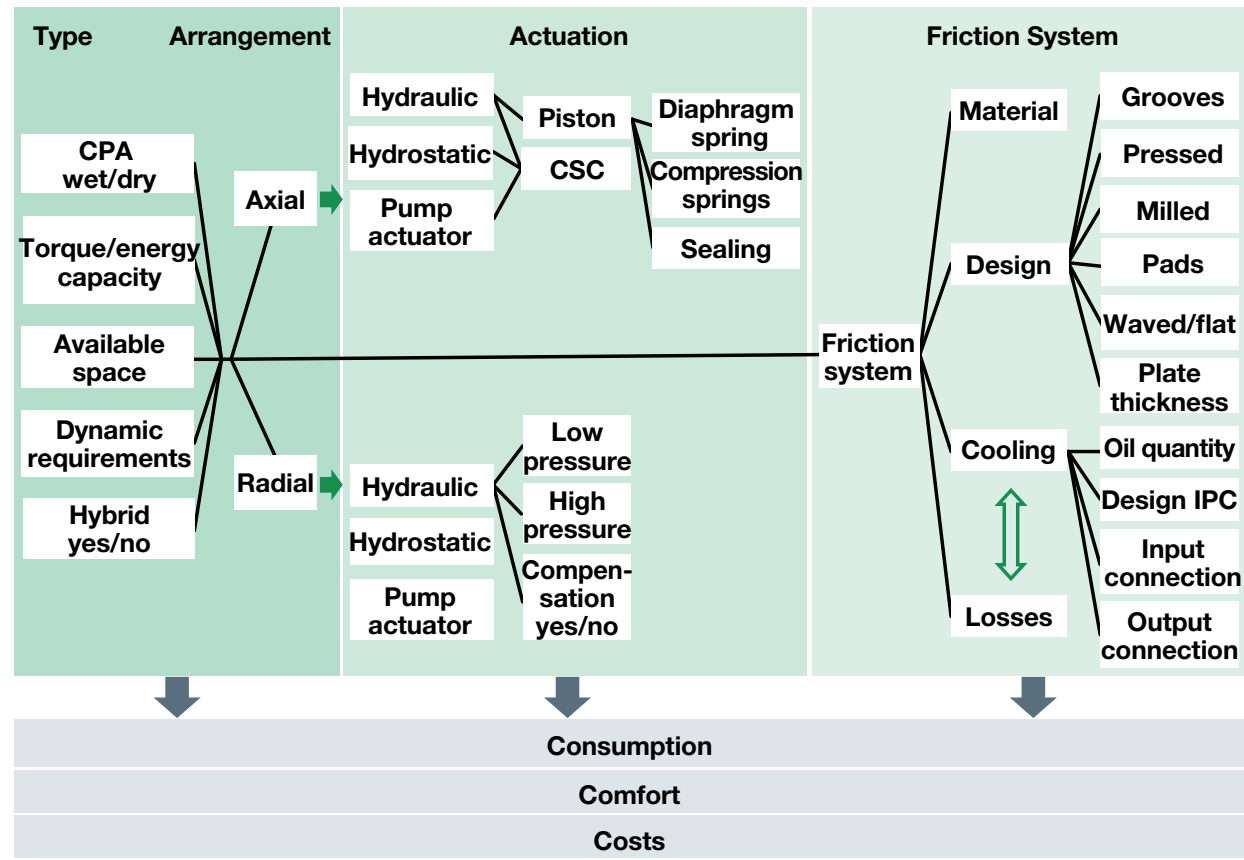

Figure 1 Wet double clutch system

Schaeffler Technologies GmbH \& Co. KG, Solving the Powertrain Puzzle,

DOI 10.1007/978-3-658-06430-3 17, (C) The Author(s) 2014 
components were examined in detail and developed accordingly.

The tribological system (comprising a friction plate, steel plate and oil), in particular, plays a key role in the design and comfort characteristics of the clutch. In addition to examining different friction linings and friction lining technologies, geometry, grooving, as well as the distribution of cooling oil and pressure are all important. Furthermore, the gathered findings and experience will be used to develop our own linings for wet clutches.

\section{The customer's perspective}

When choosing a system, customers are faced with a whole range of difficult decisions and questions that will significantly impact the architecture of the clutch system.

a) Which engines are to be used in the future?

- Are 3- and 2-cylinder applications to be taken into account?

- Does cylinder deactivation need to be taken into account?

- What are the minimum drive speeds that should be achieved?

b) What measures are to be included to further reduce fuel consumption?

- Is hybridization of the powertrain expected?

- What are the maximum torques to be taken into consideration?

- How is the clutch to be actuated?

c) How do these measures impact on fuel consumption and driving performance?

d) What kind of oil and oil flow rate is required for cooling and, if necessary, for actuating the double clutch?
All of these functions have a crucial impact on the consumption, comfort and, of course, the cost of the system.

\section{Design}

\section{Clutch}

According to the specified space requirements, wet double clutches can be designed in radial and axial forms, with the different designs offering various advantages depending on the application.

The axial design allows moments of inertia to be kept to a minimum in relation to the transmission input shaft. It also provides the option of cooling both clutches independently of each other, thereby continuing to reduce the drag losses of the open clutch. The radial construction continues to represent the preferred solution for front transverse and rear longitudinal applications. The trend towards minimizing the inertia of masses could also make axial solutions more interesting for transverse applications.

There is the option of combining both systems with a centrifugal pendulumtype absorber in the wet area; this can help to further reduce fuel consumption and improve comfort. Figure 2 shows clutches for $180 \mathrm{Nm}$ with extremely compact dimensions and a centrifugal pendulum-type absorber integrated into the clutch. In some cases, using a centrifugal pendulum-type absorber even reduces the total space required, as an additional secondary mass on the DMF is not required. 
Max. Input torque $180 \mathrm{Nm}$
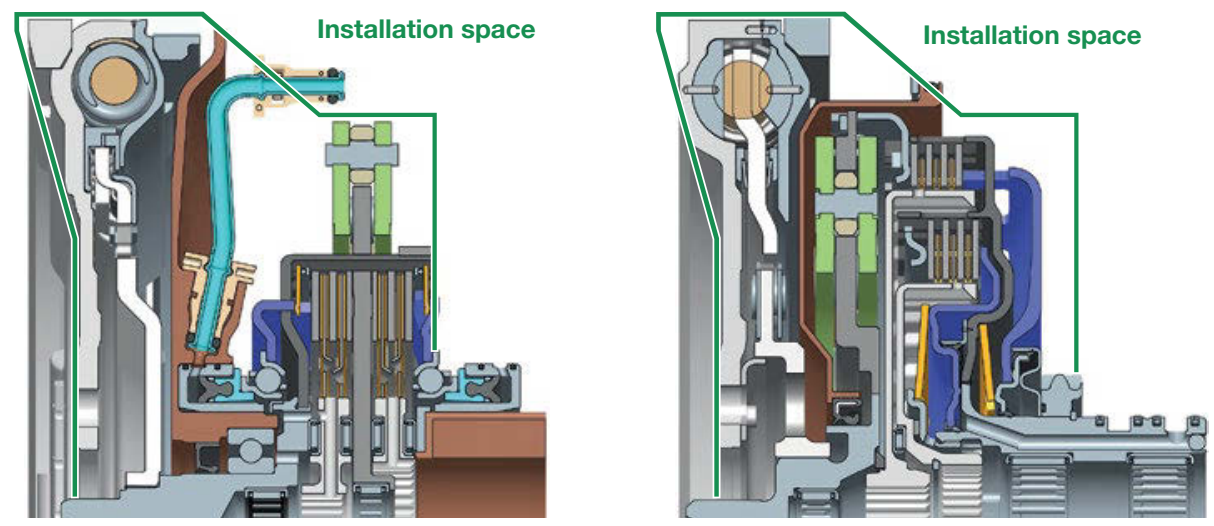

Figure 2 A wet double clutch for $180 \mathrm{Nm}$ in axial and radial design with a centrifugal pendulum-type absorber [2]

\section{Centrifugal pendulum-type absorbers in wet area}

Using the centrifugal pendulum-type absorber (CPA) in the double clutch enables a substantial reduction of the drive speeds, there- by also reducing fuel consumption. Figure 3 shows the quality of decoupling by the centrifugal pendulum-type absorber, on the basis of excitation by a 3-cylinder engine. The engine can be operated at very low engine speeds, without compromising on comfort.

Due to the minimal additional space required, these kinds of solutions in the wet


Figure 3 Quality of vibration decoupling with 3-cylinder engine [2] 



\begin{tabular}{|l|c|c|}
\hline & + CPA & + J_sec \\
\hline Total secondary moment of inertia in $\mathbf{~ k g m}^{2}$ & 0.040 & 0.105 \\
\hline Weight difference in $\mathrm{kg}$ & 0 & +7.0 \\
\hline Additional axial length required in $\mathrm{mm}$ & 0 & 10 \\
\hline Distance difference after $4 \mathrm{~s}$ in $\mathrm{m}$ & 0 & -1.9 \\
\hline Time to accelerate from 0 to $100 \mathrm{~km} / \mathrm{h}$ in \% & 0 & +4.1 \\
\hline
\end{tabular}

Figure 4 Double clutch with and without centrifugal pendulum-type absorber: differences in the mass moment of inertia, in weight, in the axial length as well as in the driving performances

area can be easily implemented in almost any transmission. Using the pendulumtype absorber enables the total mass of the clutch and DMF, and therefore also of the inertia of masses, to be reduced, in addition to reducing the minimum drive speeds. For smaller engines in particular, the result is improved dynamics while simultaneously lowering fuel consumption. Comparing driving performances of a vehicle fitted with the appropriate equipment also documented the positive effects (Figure 4).

\section{Actuators}

The clutch can be actuated hydraulically, hydrostatically or by using pump actuators. Hydrostatic systems (HCA) offer the advan- tage that power-on-demand systems, and thus significant benefits to efficiency, can be realized. This is reflected in Figure 5: Clutch and gear actuators only contribute $4.6 \%$ to overall transmission losses in the NEDC (New European Driving Cycle). However, individual operating points need be to examined in much greater detail, such as if the clutch is to be used at low temperatures (from $-20^{\circ} \mathrm{C}$ to $-30^{\circ} \mathrm{C}$ ).

The hydraulic systems offer the advantage of high power density, but a permanent power input is also required.

A combination of clutch, CSC (concentric slave cylinder), hydrostatic clutch actuator (HCA) and gear actuator represents the best version in terms of energy. In order to prove this, consumption simulations will be performed using a detailed model of the powertrain, so that the efficiency of individual components can be evaluated. Furthermore, different concepts can be evaluated, such as concepts 


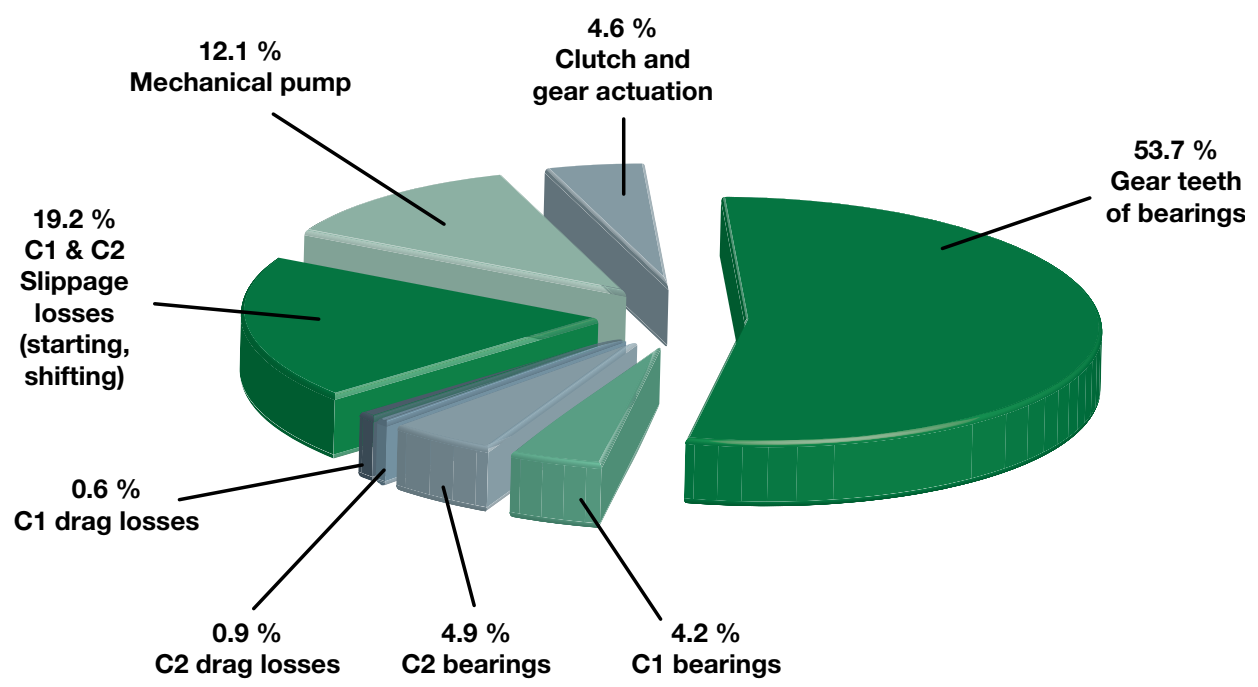

Figure 5 Distribution of transmission losses in the NEDC for 180 Nm DCT with HCA and gear actuators

for the low-pressure pump used and the operating strategy for clutch and bearing lubrication.

The result of the NEDC simulation (Figure 5) shows that DCT-specific losses from actuators and clutch drag losses can be reduced to a fraction of the mechanical losses.

Figure 5 also shows that the lowpressure pump driven on the primary side that is used as a basis for the calculation indicates a share of $11.5 \%$ of total losses. It is possible to significantly reduce this share of total losses to around $3 \%$ if the pump driven by the engine speed is replaced with an electrically driven one (Figure 6). The reason for this is the small time portion of approximately $9 \%$ when starting or shifting in which a higher cooling oil flow is required during driving mode for the slipping clutch. In contrast, the pump can be operated at a lower speed and therefore lower drive power with considerably higher time portions of approx. $70 \%$ (start-stop system taken into account here) in order to provide the minimum oil quantity for the non-actuated clutch and bearings.

One aspect already mentioned has a positive impact on both pump concepts: The optimized groove geometry of the lining plates results in an improved oil distribution requiring a significantly smaller quantity of cooling oil. The pump can therefore be designed for a smaller flow volume.

\section{Dynamics}

The previous sections looked at the benefits of hydrostatic control with HCA, in particular at the small share of clutch actuators in the overall transmission losses in the NEDC. In this section, the dynamic behaviour of the line, comprising a hydrostatic clutch actuator, the CSC and 




Figure 6 Measuring the dynamics of clutch C1: the standardized parameters are shown - actuator position, actuator pressure as well as the torque transmitted by clutch $\mathrm{C} 1$.

the clutch, is explained using measurements.

Figure 6 shows the measured profile for operation of clutch $\mathrm{C} 1$. The parameters shown are standardized to the respective maximum values to enable uniform presentation. Starting from the initial value of approximately $21 \%$ the actuator position starts to change after $50 \mathrm{~ms}$. On account of the clutch characteristic curve, the clearance must first be overcome until the touch point (TP) is reached. From this point onwards, the actuator pressure increases significantly. With a small time delay the torque transmitted by clutch $\mathrm{C} 1$ also increases. The actuator position reaches the target value (100 \%) after approx. $200 \mathrm{~ms}$, which in this case is equivalent to the maximum driving torque according to the design. It takes approx. $100 \mathrm{~ms}$ until the maximum pressure is reached. The maximum torque is transmitted as early as $120 \mathrm{~ms}$ after pressure has started to build up. The measurement data relates to a $550 \mathrm{Nm}$ DCT. For smaller clutches and therefore lower actuation forces, the dynamics can be increased further.

In order to keep the time difference between the actuator position starting to change and reaching the required torque as small as possible in a real driving situation, the actuator position is not moved to $0 \mathrm{~mm}$ in a waiting position (WP) of the non-actuated clutch; instead, it is moved in the clearance range just underneath the TP. The friction system is described in greater detail in one of the following chapters, which also lists the measures designed to minimize drag losses in the clutches. Using these measures, it is possible to keep the spacing of the waiting position from the TP and the drag torque through the non-actuated clutch as small as possible. Doing so achieves short actuation times for adjusting a required torque.

\section{Axial or radial design}

Double clutches in axial and radial designs are compared in detail in this section. This is again based on the NEDC simulation, and the share of the individual components in the overall transmission losses are discussed. According to Figure 2, the axial design comprises two wet clutch release bearings with CSC. Rotary connections with sliding ring seals are taken into consideration for the radial design concept. However, in principle, a radial double clutch can also be actuated via a CSC.

Due to the geometric ratios, the drag torques of $\mathrm{C} 1$ and $\mathrm{C} 2$ and the resulting shares in the overall transmission losses (Figure 8) are approximately 1 percentage point smaller for the axial arrangement than for the radial concept. The drag torques are calculated based on the measurements on the NEDC operating points. The measure- 
ments regarding drag losses of various plate geometries are described in greater detail in a subsequent section.

\section{Diaphragm springs or compression springs}

Using diaphragm springs for opening the clutch pack represents a space-saving alternative to spiral springs. The question that therefore needs to be asked is how do these elements influence of the clutch hysteresis. As part of this question, a solution using diaphragm springs does not automatically lead to higher hysteresis values. The example shown indicates that, with the right design of the diaphragm spring, contact surface and overall system, it is possible to achieve hysteresis values comparable to those of compression spring solutions.

\section{Rotary connection or CSC}

Rotary connections are used in many of today's double clutch transmissions and provide a robust solution in conjunction with hydraulic systems. Wet clutch release bearings with CSC can be used as an alternative; the losses of these bearings are much lower in comparison with the rotary connections. Applications up to $700 \mathrm{Nm}$ are currently in the development phase.

Furthermore, the bearing concept of the axial arrangement with a deep groove ball bearing, four axial needle roller bearings and two clutch release bearings for CSC indicate a benefit of more than $3 \%$ points in comparison with sliding ring seals. Again, measured values are converted into NEDC operating points. Furthermore, the CSC offers the option of minimizing geometrical deviations in the clutch to be similar to the dry system.

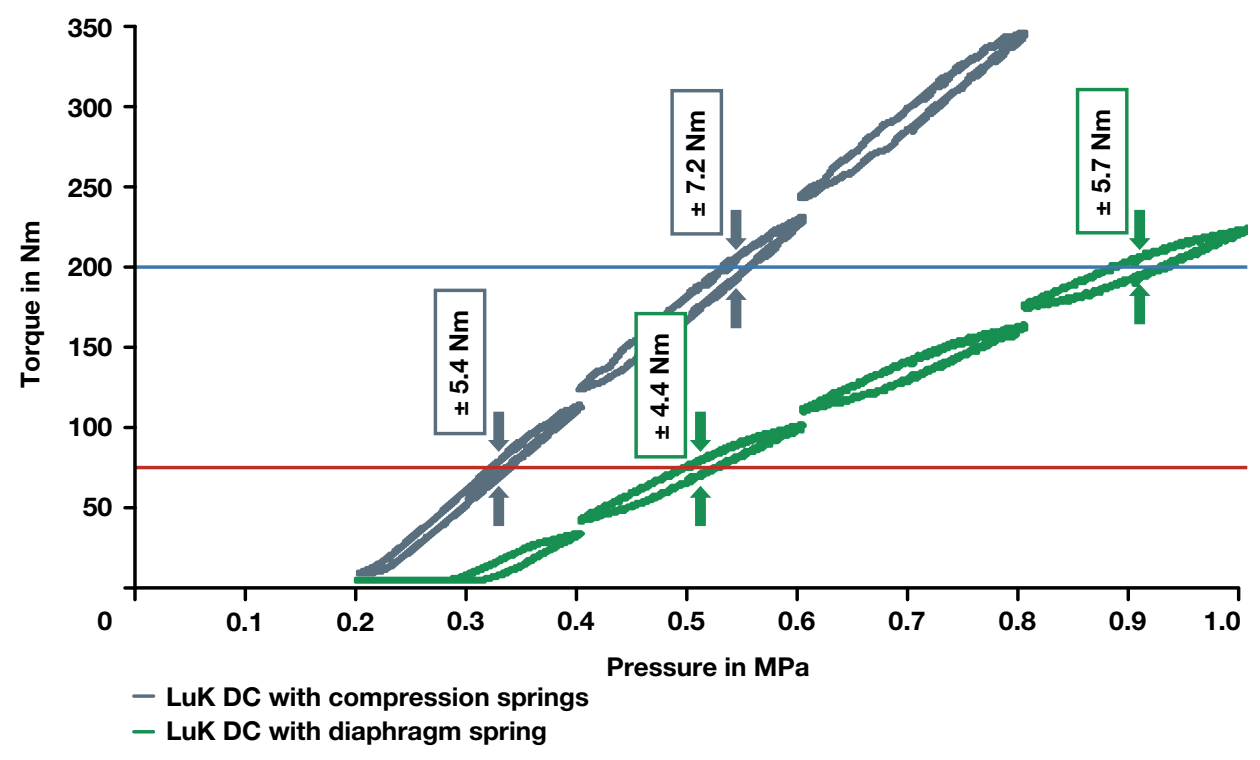

Figure 7 Comparing the clutch hysteresis 

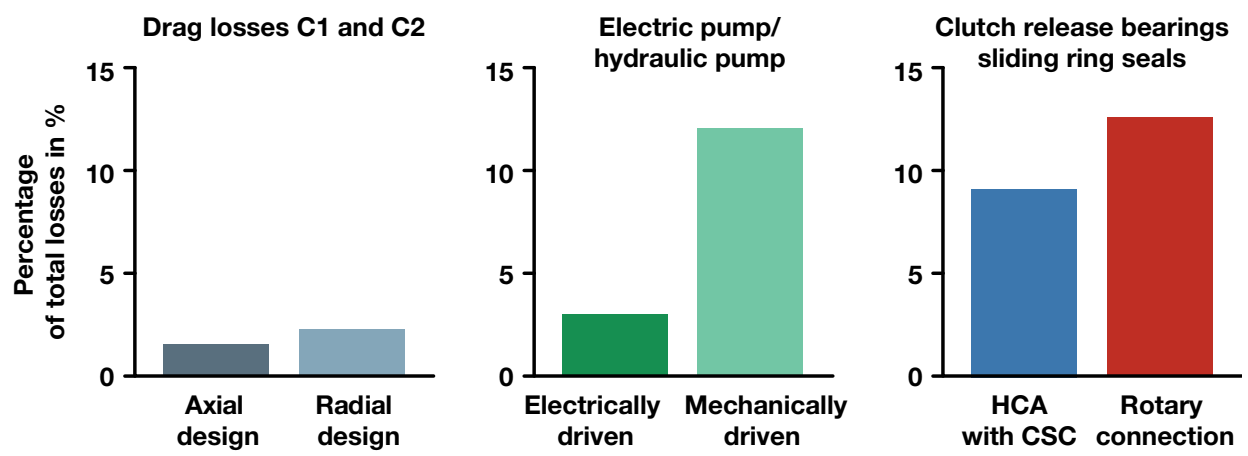

Figure 8 Comparison of axial and radial designs: share of C1/C2 drag torques, as well as of the bearings (CSC) and the sliding ring seals for rotary connections in the overall losses in the NEDC

\section{The friction system}

\section{Design}

Waves and grooves play a key role in how the wet clutch works. The manufacturing method also significantly influences the friction value structure and the resulting drag losses. In addition, the lining's geometric characteristics play an essential role in the uniformity of torque transmission. Depending on the volume flow, there is a considerable increase in the clutch drag torques.

The aim of the development phase is therefore to minimize the cooling oil volume flow, optimize the groove geometry and ensure the correct wave of the plate.
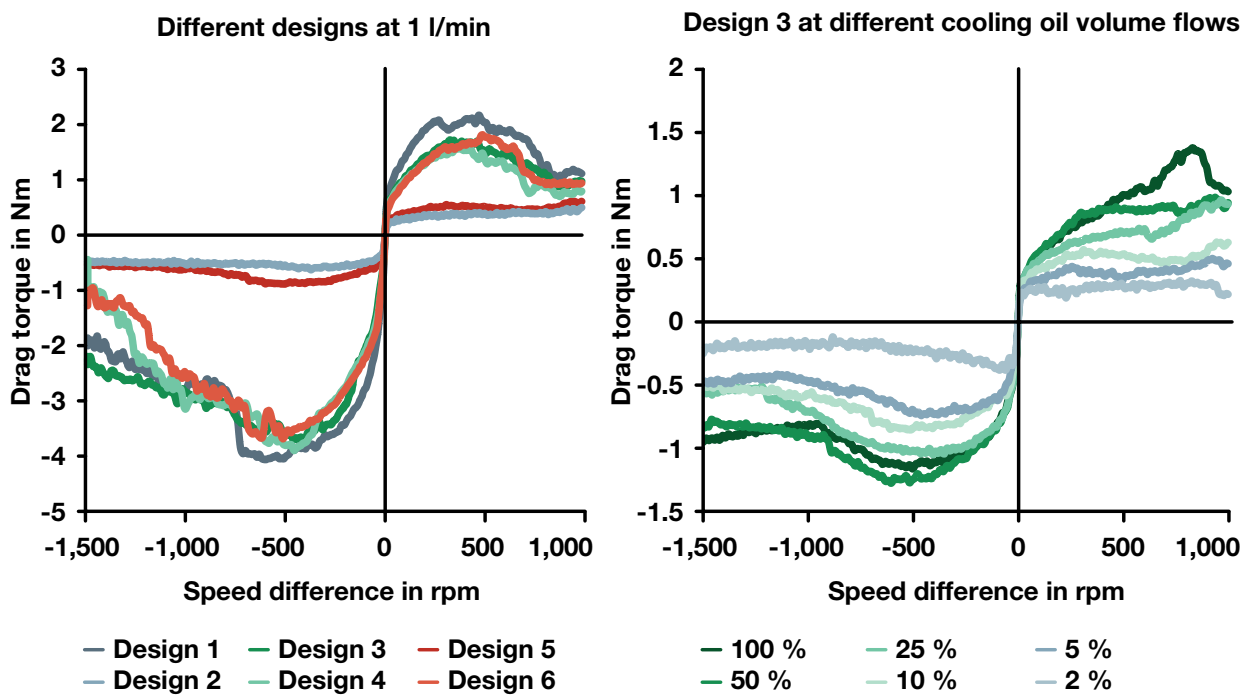

Figure 9 Impact of the plate geometry on drag losses 



Figure 10 CFD analysis and comparison with test results

\section{Cooling}

The cooling effect is heavily influenced by the connection of the friction plates (primary or secondary side), the layout of the bores in the inner plate carrier and the lining groove geometry.

By using CFD analyses, these effects can now also be demonstrated with simulations. Figure 10 shows the influence of the bore pattern in the inner plate carrier and the influence of the groove geometry.

Discoloration of plates clearly shows the poorly cooled areas. Good consistency can be seen between the simulation and the thermal load of the plate.

Furthermore, the thickness of the plates must be optimized depending on the specific application.
Stability of friction value in endurance test

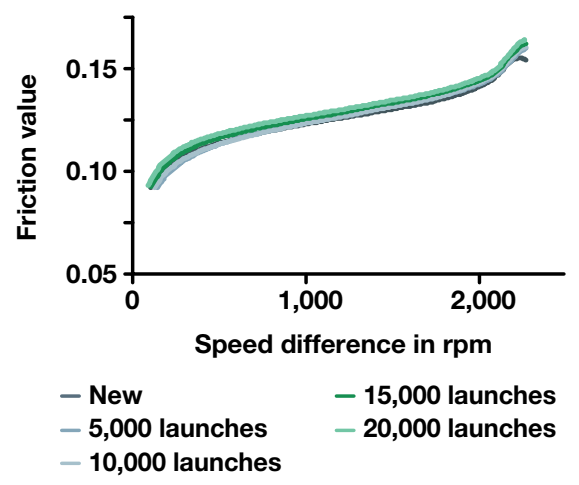

Friction plate after $\mathbf{2 0 , 0 0 0}$ hill starts under full load

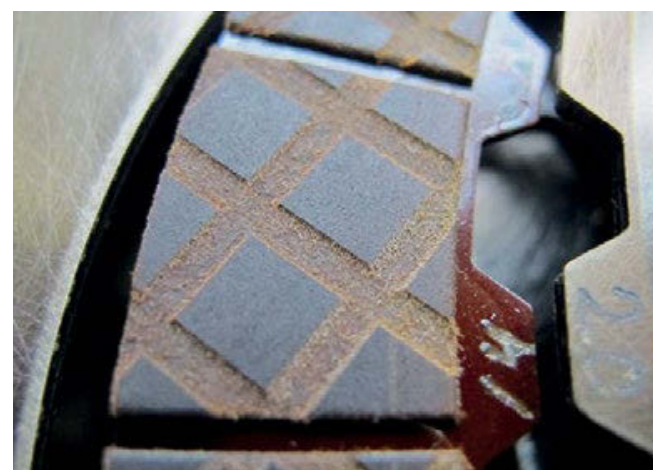

Figure 11 Endurance test with LuK friction lining: 20,000 hill starts under full load 


\section{LuK lining}

The friction plate really comes into its own within the tribological system. The plate has a significant impact on the friction value, the friction value gradient, the wear behaviour and the thermal capacity of the clutch, which is why LuK pushed the development of its own double clutch linings. The suitability of LuK lining in functional and endurance tests has since been demonstrated (Figure 11). The characteristic curves clearly show that the friction value gradient is following a very strong and stable, positive course. It should therefore be possible to reliably rule out clutch judder due to the friction value.

Running in parallel with the development of wet double clutch linings is the advancement of their industrialization. tently optimizing damping components, the clutch and the clutch and gear actuators.

LuK friction linings for wet double clutches have demonstrated their suitability for DCTs.

In combination with the software, it is possible to find special solutions that improve the driving performance, significantly increase driving pleasure and further reduce fuel consumption for every customer.

In conjunction with modern hybrid systems, future powertrains can therefore be realized with a high level of comfort and minimum fuel consumption.

\section{Literature}

[1] Zink, M.; Wagner, U.; Feltz, C.: Double Clutch Systems - Modular und Highly Efficient for the Powertrain of Tomorrow, $10^{\text {th }}$ Schaeffler Sympoisum, 2014

\section{Wet double clutch}

[2] Baumgartner, A.; Lauinger, C.; Lorenz, E.; Fischer, N.; Goetz, A.; Krause, T.: Reducing $\mathrm{CO}_{2}$ emissions with wet double clutch transmissions with a simultaneous improvement in comfort and driving dynamics. VDI Getriebe in Fahrzeugen, 2013

\section{The modular design}

Wet double clutches are now available in in radial and axial designs for torques between $100 \mathrm{Nm}$ and $3500 \mathrm{Nm}$ (Figure 12). The double clutches can be combined with centrifugal pendulum-type absorbers as an option.

The efficiency of the overall transmission can be ensured by consis-
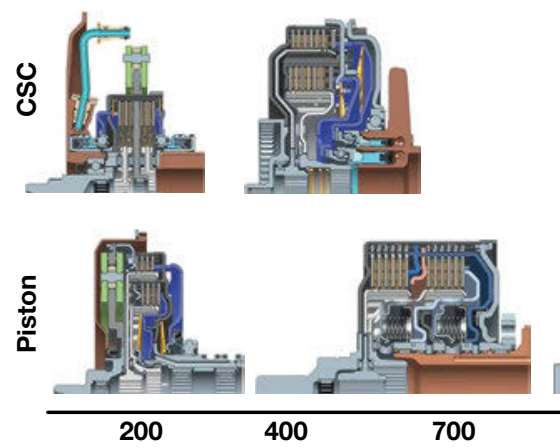

400

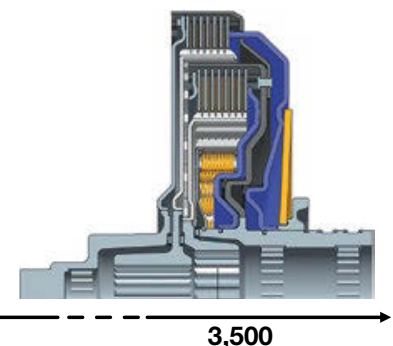

Engine torque in $\mathrm{Nm}$

Figure 12 Modular design

Open Access. This chapter is distributed under the terms of the Creative Commons Attribution Noncommercial License, which permits any noncommercial use, distribution, and reproduction in any medium, provided the original author(s) and source are credited. 